\title{
Volume Rendering Phenotype Differences in Mouse Placenta Microscopy Data
}

\author{
Richard P. Sharp* \\ Alain de Bruin"
}

\author{
Randall Ridgway ${ }^{\dagger}$ \\ Raghu Machiraju**
}

\author{
Kishore Mosaliganti \\ Kun Huang
}

\author{
Pamela Wenzel ${ }^{\S}$ \\ Gustavo Leone
}

The Ohio State University

\begin{abstract}
In this work we present an application problem of examining phenotype differences in wildtype and retinoblastoma $(R b)$ knockout specimens. The lack of the $R b$ gene causes tissue infiltrations into critical sections of mouse placenta that lead to fetal death.

This paper describes our method for volume visualization of tissue level intermixing at a microscopic scale for both wildtype and $R b$ knockout specimens. Our technique combines non-trivial registration techniques, a two-level Bayesian classifier, and a volume rendering step. Our final volume renderings show tissue intermixing differences between both wildtype and $R b$ knockout specimens that are not obvious from examining the two dimensional image stack alone.

CR Categories: I.3.6 [Computing Methodologies]: Computer Graphics-Methodology and Techniques; I.3.7 [Computing Methodologies]: Computer Graphics-Three-Dimensional Graphics and Realism; I.3.8 [Computing Methodologies]: Computer Graphics-Applications
\end{abstract}

Keywords: volume rendering, histology, biological segmentation

\section{Application Problem}

In this paper we address the problem of visualizing datasets of histologically stained microscopy slides. In particular, mouse placenta with and without the retinoblastoma gene $(R b)$. This gene is one of the first to be associated with a specific cancer (retinoblastoma) and has been studied extensively in both human and mouse cell models [8].

Mouse placenta are routinely used in these studies and are composed of three distinct layers: the labyrinth, spongiotrophoblast, and glycogen layers. Figure 1 shows a sample slide with the approximate labyrinth, glycogen, and spongiotrophoblast layers marked in red, yellow, and blue, respectively.

Recently, it has been shown that inactivation of the $R b$ gene $\left(R b^{-}\right)$in a mouse embryo results in morphological changes in the placenta, including reduction in vascularity of the labyrinth layer. It is postulated that a decrease in vascularity contributes to fetal death at 14.5 days of gestation [15]. Cancer geneticists have immense interest in studying the three-dimensional structural changes that occur by the inactivation of the $\left(R b^{-}\right)$gene. More specifically,

\footnotetext{
*sharpr@cse.ohio-state.edu

†ridgway.13@osu.edu

${ }^{\ddagger}$ mosaligk@cse.ohio-state.edu

\$Pamela.Wenzel@osumc.edu

Itpan@bmi.osu.edu

\|Alain.debruin@osumc.edu

**raghu@cse.ohio-state.edu
}

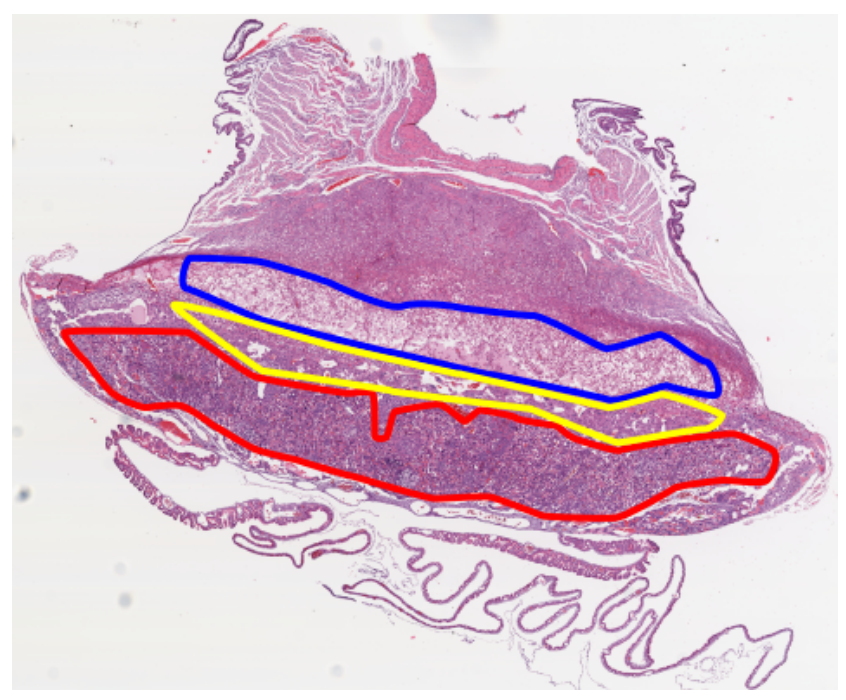

Figure 1: An example of one of the $R b^{-}$histological mouse placenta slides. The red, yellow, and blue markings show the approximate locations of the labyrinth, glycogen, and spongiotrophoblast layers, respectively. Tissues not marked are considered "maternal tissue".

the infiltrations of the spongiotrophoblast layers into the labyrinth are of great interest. These infiltrations reduce the surface area of the labyrinth resulting in fetal death. Our cancer geneticist collaborators are interested in viewing the infiltrations in their complete three-dimensional form. Figure 2 shows a sample volume render of the cropped data with opacity derived off the luminance of the slides.

Our goal is to reconstruct and visualize the three-dimensional intermixing of tissue types of specimens given their sectioned sets of RGB histology slides. This problem is similar to that which radiologists experienced a decade ago in visualizing $\mathrm{CT}$ and MR data. However, unlike CT and MR images, our data is not co-registered, is difficult to segment, and requires the highlighting of sub-tissue level features in 3D. We explain these challenges in detail later in Section 1.3. Although the data presented in this paper is that of mouse placenta, these techniques could be applied on other types of histologically stained slides.

\subsection{Related Work}

Our work focuses on taking a set of microscopy histological slices, reconstructing and realigning the slides into volumes, segmenting the volume and finally volume visualizing the characteristic intermixing of different tissue types in 3D. Below we discuss previous work that have considered similar problems in registration, segmentation and $3 \mathrm{D}$ reconstruction.

Johnson and Christensen [6] present a hybrid 
(a)

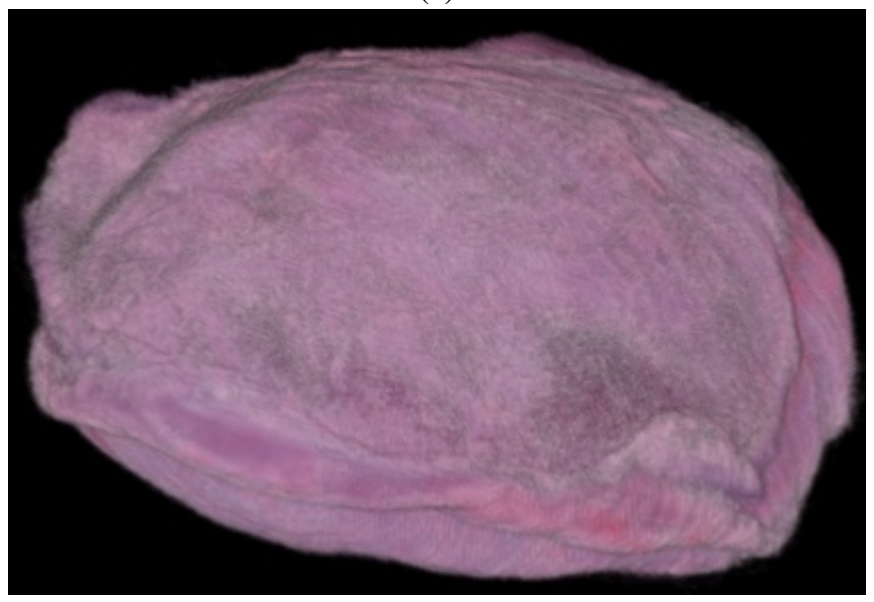

(b)

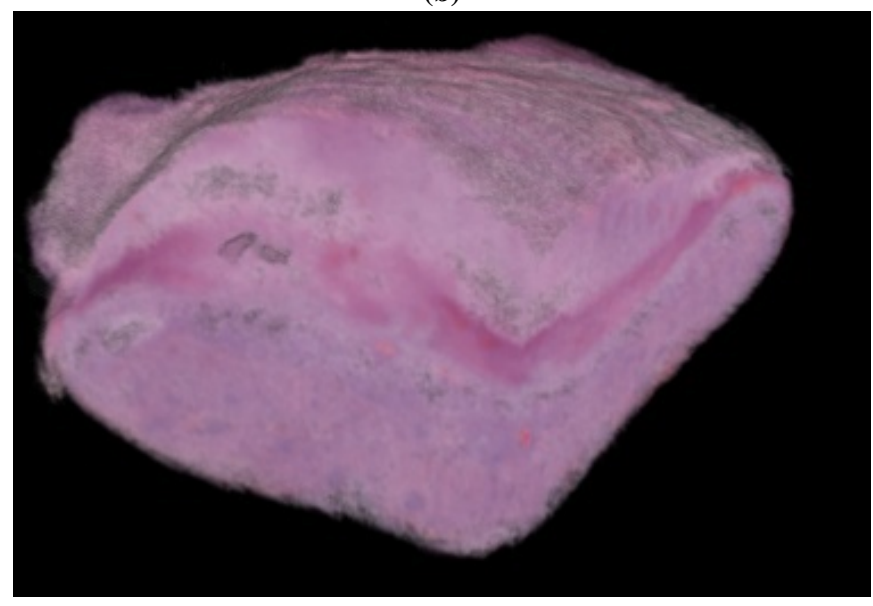

Figure 2: (a) Sample volume rendering of a wildtype mouse placenta, original size is approximately $1 \mathrm{~mm}^{3}$. (b) The volume has been cropped so the internal structure of the placenta is exposed. The tissue specific structures (labyrinth/spongiotrophoblast mixing) are difficult to see in this view.

landmark/intensity-based deformable registration technique. The approach uses an iterative process of first registering landmarks independently of intensity, then attempts to minimize image intensity difference independent of landmarks. While this approach is useful for mapping $2 \mathrm{D}$ brain sections to brain-atlases, this approach would not work well on our data. First, it is not obvious from the unclassified data what constitutes a landmark point, let alone the challenge of manually marking landmarks for thousands of placenta slices. Furthermore, we are interested in the original 3D shape of the placenta. Deformable registration will corrupt the overall $3 \mathrm{D}$ shape and possibly the intermixing of the tissues.

Arganda-Carreras et al. present a method for automatic registration of histology sections in [1]. The authors present two techniques for non-deformable registration using Sobel transforms and segmentation contours. Although we are solving a very similar problem our data does not have well defined contours on a slice by slice basis. Thus, contour based registration techniques fail on our dataset.

Teverovskiy et al. [14] develop a system to identify epithelial nuclei present in prostate tissue. Their technique uses a regiongrowing algorithm to find contiguous regions based on color similarity and shape regularity. Then, they perform a coarse segmentation based on staining color differences. Finally, a refined segmentation is used to separate regions of nuclei clumped with cytoplasm. Although initially we do pixel level classification, our final segmentation differs from Teverovskiy et al. since we are interested in classifying entire tissue regions rather than locations of sub-tissue structures.

Petushi et al. [11] segment specific tissue types from histology slides. Their technique is based on a hybrid method that combines optimal adaptive thresholding with local morphological opening and closing operations. Unfortunately this method is only good at identifying sub-tissue structures, not entire tissue sections.

Mojsilovic et al. [7] present a technique for reconstructing the 3D surface of brain tumors from histological slices. Their goal is to identify tumor boundaries, isolate the proliferating tumor cell and segment the tumor into regions based on the density of proliferating cells. Our work differs in part due to the large datasets we work with. Usually a dataset is on the order of 800 slides. Furthermore, Mojslivoic et al. is concerned mainly with reconstructing an approximation to the boundary of the tumor. We are interested in the internal high frequency infiltrations into the labyrinth layer, which requires higher quality segmentation and registration techniques as well as volume rendering to visualize the intermixing.

Ourselin et al. also present a technique for 3D reconstruction based on histology slices [10]. Their work mostly focuses on blockmatching registration techniques, which are demonstrated on both rat and rhesus monkey brain slices. Our work is similar in the sense that the data must be registered, however our microscopy images are much nosier and have more artifacts than brain histology slices. Furthermore our goal is to identify intermixing of layers, not just reconstruct the three dimensional shape. Finally, Clendenon et al. [2] have developed a volume renderer, Voxx, for volume rendering confocal and two-photon fluorescence microscopy images. Essentially the system renders sets of images in back-to-front order and combines them using alpha blending.

\subsection{Approach}

In this article we discuss the issues and solutions we developed to assess the volumetric changes of various tissue types in both wildtype and knockout $R b^{-}$mouse placenta. The overall technical steps for this process are as follows:

1. Segment each image into regions corresponding to the three tissue types.

2. Register the serial sections of the given placenta to reconstruct a virtual placenta.

3. Build three-dimensional models of each of the tissue layers by aligning the segmented images using the information collected during the registration phase.

4. Volume render the segmented volume using standard "off the shelf" algorithms.

Effectively segmenting the microscopic image slices is more challenging than segmenting images from $\mathrm{CT}$ and MR. Usually regions of interest (ROI) in CT or MR data consist of constant or smoothly varying scalar values. However, the ROIs in the placenta data (labyrinth, spongiotrophoblast, and glycogen) consist of a non-uniform distribution of cellular material (nuclei, cytoplasm, red blood cells, and vacuum). Boundaries are not well demarcated and every voxel or pixel suffers from a partial voluming effect. As 
shown later in the case of segmenting mouse placenta, we utilize several image features including pixel color value, local texture, and high-level features including count of white vacuoles within a region in a probabilistic segmentation framework.

Once the volumes are segmented and registered, we demonstrate that traditional volume rendering techniques can be used to visualize the resulting layers and the extent of their intermixing. However, rather than render the RGB data directly as in Figure 2 we take the approach of rendering the segmentation labels with fuzzy boundaries to reflect the nature of the ROI's physical boundaries.

\subsection{Challenges from Histological Data}

Volume visualization of structures in placenta data is problematic given the many artifacts introduced by the manual nature of tissue preparation. Aside from the standard registration and segmentation challenges the following artifacts introduced by the manual acquisition required additional preprocessing and modifications to the traditional rendering pipeline:

- Damaged and missing sections: During slicing and mounting, sections are occasionally torn, folded, or discarded entirely. By plotting tissue areas versus slide number we observe outliers, which automatically identify most of the damaged slides for removal.

- Staining variations: Differences in section thickness, staining duration, and stain concentration results in color variations in histologically stained specimens. Tissue boundaries are difficult to recognize when these staining variations are present.

To address this issue, our registration technique employs a clustering preprocessing step that performs well with staining variations. Before classification, histogram equalization is used to normalize the color distribution across slides. Then, during the volume rendering step the RGB data is re-colored based on the tissue type. Therefore, the variations from slide to slide have little effect on the entire rendered volume.

- Yolk-sac: Nearly every slice of the placenta contains yolksac tissue (the wispy features near the bottom of the slide in Figure 1). Since the presence of yolk-sac tissue can significantly distort during the section mounting process, we find that this tissue is oriented differently on nearly every slide in the volume. This complicates the registration process since the yolk-sac cannot be rigidly registered to reconstruct its original three-dimensional shape. Attempts at automatic removal of the yolk sac were not successful due to its complex and random structure across slides and across specimens. Instead we manually remove the yolk-sac from every slice before the register and volume render step.

In the next section we will discuss our rendering pipeline in detail while Section 3 contains our results, and Sections 4, and 5 include our future work and conclusions, respectively.

\section{Methods}

Our goal is to visualize in three-dimensions critical tissue structures for the purposes of examining the tissue intermixing in both the wildtype and knockout $R b^{-}$placenta types. As mentioned previously, our method first identifies tissue types on a per-slide basis, aligns the RGB slides and probability maps through an enhanced registration algorithm, then uses the classification to render the tissue structures directly in RGB slides. We will describe the details of this method in the following paragraphs.

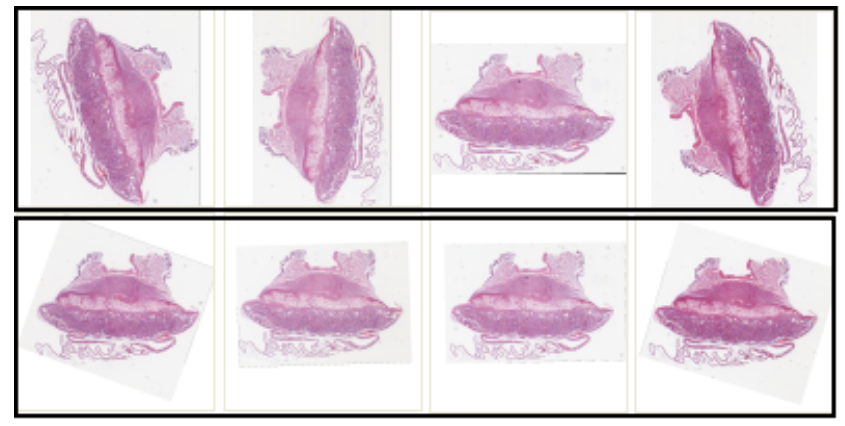

Figure 3: The top sequence shows the initial random orientation of the placenta images. The bottom sequence shows the resulting PCA-aligned images.

\subsection{Preprocessing}

Before we begin tissue classification, we first apply several preprocessing steps. First we subsample the images from $15 K \times 15 K$ pixels to $3 K \times 3 K$ prior to segmentation. The subsampled output allows us to avoid the additional complexity of out-of-core or distributed computations.

\subsection{Registration}

As we described previously in Section 1.3, microtoming introduces large numbers of artifacts into the slides. In this section we briefly describe our four step registration pipeline for the placenta data. The details of this algorithm can be referenced in [12].

In this stage we first identify defective placenta slices by plotting tissue area versus slide number for the entire dataset. The resulting plot shows discontinuities where damaged sections exist, which we then discard. Due to the large number of slices in the dataset, missing slides are not noticeable in the final visualization. If these defective slides are not removed the global registration is skewed since it is impossible to align a good slide with one that is damaged.

In the second step we detect the tissue region in the original slide so that our registration step is not confused by background artifacts. The tissue region is defined as a binary mask that removes the background for the last two steps of our outline. To detect the tissue region we use a close variant of the $k$-means [12] clustering approach with since it performs well in the presence of luminance gradient, background noise, and staining variations.

In the third step we perform an initial alignment of the placenta slices for the final registration. We chose to use principal component analysis (PCA) based alignment since mouse placenta sections are typically elongated in shape (high width to height ratio). Therefore, it performs well when estimating the orientation of the overall tissue shape. These estimates of slide orientations are used to initialize registration that in turn restricts the transform search space.

In the final step we perform a four stage registration of the placenta slides. In the first step we transform a designated moving image slide (as opposed to a reference image slide), which is resampled onto a grid. Next, we compute a mutual information-based metric on the quality of fit between the stationary and transformed images. If the metric is similar to a prior value, we stop the iterative process, else we refine the transform and start again. Figure 3 shows the results of our registration algorithm run on four sequential images. 
(a)

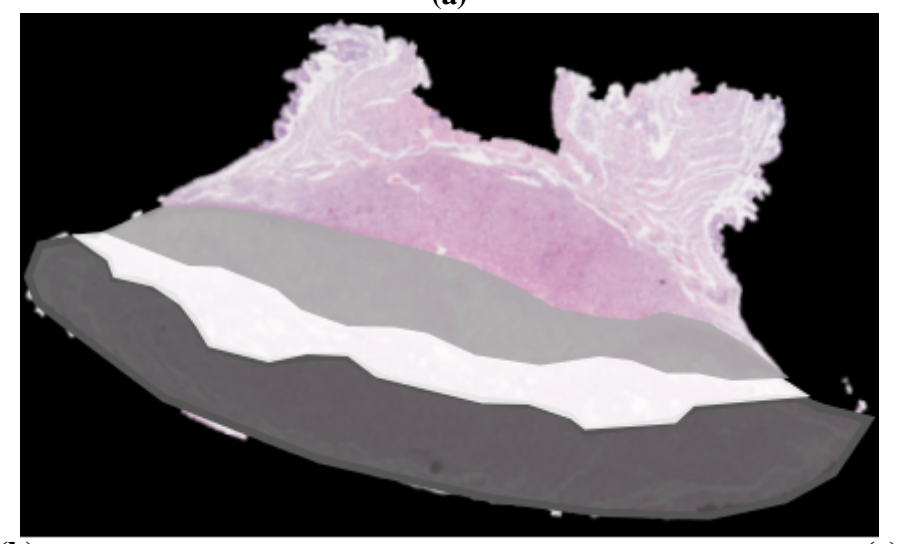

(b)
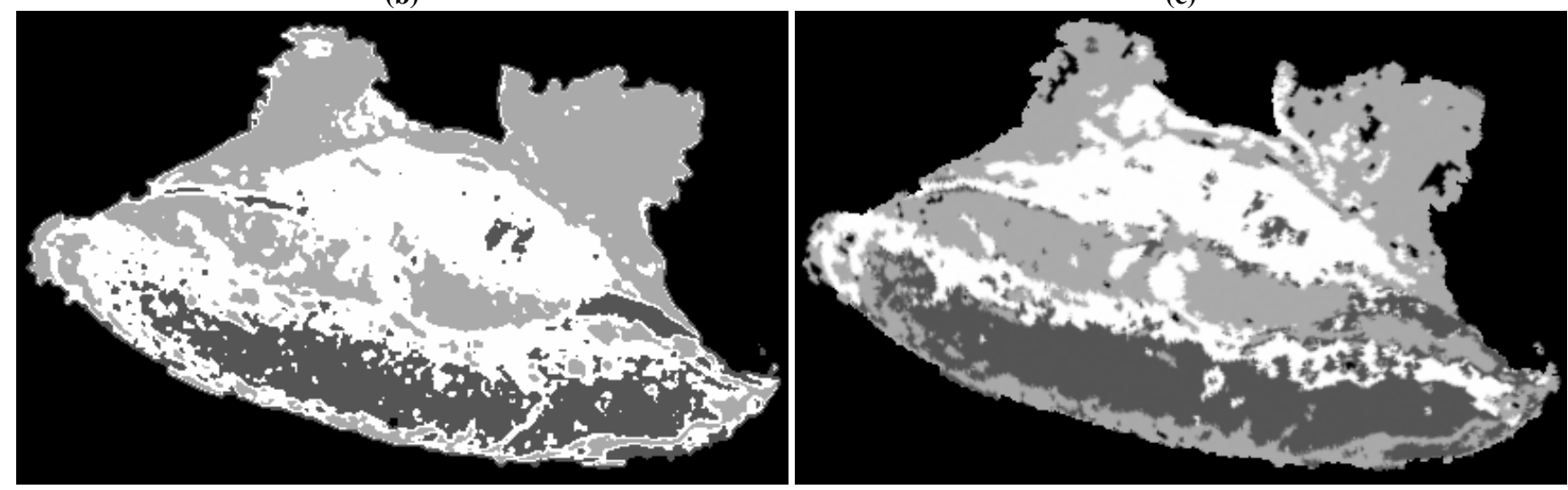

Figure 4: Figure (a) shows a placenta slice which has been hand segmented to show labyrinth (dark gray), glycogen (medium gray), and spongiotrophoblast (white). Figure (b) shows the same placenta segmented with a $k$-means filter, while Figure (c) shows the result of our Bayesian classifier used in this work.

\subsection{Tissue Classification}

When we classify the placenta, we focus on three different tissue types in the ROI: labyrinth, glycogen, and spongiotrophoblast. Other tissues such as maternal and yolk-sac are excluded from the ROI. To handle the artifact from non-uniform color intensity variation, we employ a histogram equalization process where we consider an image with an average color distribution (as determined by visual inspection) then normalize the data to that color distribution. Since the background pixels tend to vary more than the placental pixels we exclude them through luminance thresholding.

Further, the tissues in the histology images are not well demarcated and possess an inhomogeneous internal structure. Standard image segmentation techniques often fail to create any reasonable segmentation of these tissues; instead we present a probability framework to determine the probability that a certain sample is of a particular tissue. However, the framework must first be trained before it can be applied to actual test data. We generate our training data by selecting sample windows from a priori known tissue types and use them to generate a series of feature vectors on the samples. The feature vectors are then used to estimate a probability density function (PDF) for the features for all tissue types. We use these probabilities to classify each tissue type by selecting the class with the maximum a posteriori probability.

The Bayesian probabilistic approach has proved to be a very powerful tool in image segmentation elsewhere. It is particularly effective in segmenting complicated images with multiple textures such as the images discussed in this paper. We also examined using a decision tree based approach. Unfortunately the decision tree based approach required many manual tweaks per slide to achieve a quality segmentation. Thus, the Bayesian framework is preferable since it only requires per-dataset training data which is much easier to generate that then per slide tweaks in the decision tree approach. We have also attempted to use a $k$-means classification routine but have found that it gives poorer results than our Bayesian routine. See Figure 4 for a visual comparison of the results of these classification scheme.

An essential component of our segmentation is pixel-level classification. Once again, we have experimented with various methods of pixel level classification including standard thresholding techniques and classifiers based on the $k$-means algorithms. In some placenta datasets these methods worked quite well, while in others many adjustments were required to they failed. Once again we found the Bayesian classifier to be more robust than other methods.

Note that we use a 2D segmentation method for two reasons: first a true three-dimensional segmentation would require expensive out of core processing due to the sheer size of the data and secondly, microtoming causes shear deformation in the slides that shifts subtissue level features such as spongiotrophoblast infiltrations. Since these shifts vary on a slice-by-slice basis a three-dimensional segmentation could potentially smooth many of these important structures away.

Figure 4 shows the result of our probability framework. The color image shows an original placenta slice which has been approximately hand segmented. The two grayscale images compare the results of a $k$-means classification algorithm with our Bayesian classification routine presented in this work. We describe overall 
structure of our classification method in the following sections. For details and validation of this algorithm see [13].

\subsubsection{Feature Selection and Feature Vector Generation}

To train our Bayesian probability framework we first decide which image and tissue level features are relevant to the classification process. Fortunately the three tissue classes show significant differences in composition of pixel values, specifically: spongiotrophoblast typically have large amounts of cytoplasm and lighter nuclei, thus appearing more homogeneous; labyrinth contains dark nuclei, red blood cells, and background from blood vessel cross sections; and the glycogen layer typically contains large amount of background, smaller cytoplasm size, and darker nuclei, in comparison to the labyrinth or the spongiotrophoblast. Given these characterizations, we choose our feature vector to include pixel level color differences, object level features (nuclei size, vacuole presence, etc.), and overall red-blood cell count.

At the pixel level we capture differences in tissue appearance with color histograms. Within a sample window, each color channel is summarized as a normalized histogram with 64 bins per channel. Then local boundaries are captured through histograms of normalized gradient magnitude of the three color channels.

Our second feature vector is an object level metric derived from vacuole and nuclei density and size. These features are important since their density is characteristic of each of the three tissue types. Our process for vacuole/nuclei identification is a three part method. First we segment background, nuclei, cytoplasm and red blood cell pixels with a naive Bayes classifier ${ }^{1}$. Secondly, background vacuoles and nuclei are identified by using a 4-connected component labeling of previously marked pixels. Finally, we build the nuclei and vacuole size histograms. Since the total number of nuclei or vacuoles is variable, the histogram bins are not correlated in this case.

The final element in our feature vector is a red pixel count, which is directly derived from the result of the pixel-level Bayes classifier. We build this by simply counting the number of red blood cell pixels in the window of consideration and add it to the feature vector as a single value. Thus, we generate a feature vector containing color, texture and object level measurements for each sample window in training and test sets.

\subsubsection{Probability Density Function of Class Features}

To compute the PDF of the class features we first assume that the components in the features vector are independent and construct the PDF for each feature component separately. Our training set consists of three classes, each with forty-eight samples. The feature PDF is then generated directly from value distributions of the forty-eight samples to model arbitrarily complex PDFs, typical in complex biological systems.

For each component in the feature vector, we generate a histogram $H$ using corresponding components from all training samples of the same class. To reduce the effect of random noise given the relatively small sample size, we smooth $H$ using a discrete Gaussian kernel three bins wide. We then normalize the smoothed histogram so that it represents the PDF of a single feature component of a class.

\subsubsection{Likelihood Computation}

After PDF have been constructed for different classes, we analyze a test image by dividing the image into sample windows and calculat-

\footnotetext{
${ }^{1}$ Nuclei, red blood cells, cytoplasm distributions and a priori probabilities for the classes were modeled by Gaussian distributions based off of a 2000 pixel training set.
}

ing each sample window's feature vector and its class likelihoods.

Assuming independence of the features, the total probability of the feature vector is the product of the individual probabilities:

$$
P(X)=\prod_{i=1 \ldots n} P\left(X_{i}\right)
$$

where $n$ is the dimension of the feature vector. The function $P\left(X_{i}\right)$ is estimated by taking the $i^{t h}$ component of the feature vector, and determining the probability in the corresponding distribution function.

\subsubsection{Bayes Decision Framework}

Our Bayesian decision framework relies on the Bayes formula for conditional probability:

$$
P(C \mid X)=\frac{P(X \mid C) * P(C)}{P(X)}
$$

where $P(C \mid X)$ is the posterior probability that a sample belongs to class $\mathrm{C}$ given a feature vector $\mathrm{X}, P(X \mid C)$ is the likelihood that a sample from class $C$ possesses all features of $X . P(C)$ is the prior probability of occurrence for class $C$, while $P(X)$ is the evidence probability of the occurrence of feature $X$ in all classes.

We classify by identifying the class to which the feature vector most probably belongs. The maximum a posteriori classifier is used to find the class $d$ :

$$
\begin{aligned}
d & =\arg \max _{i} P\left(C_{i} \mid X\right) \\
& =\arg \max _{i} \frac{P\left(X \mid C_{i}\right) * P\left(C_{i}\right)}{P(X)}
\end{aligned}
$$

It should be noted that evidence probability, $P(X)$, is essentially a scaling constant. Assuming that the a priori probabilities are uniform for all classes ${ }^{2}$, then

$$
d=\arg \max _{i} P\left(X \mid C_{i}\right)
$$

\subsubsection{Tissue Boundary Identification}

To generate the probability maps we iterate through small regions in the test image. Each sample region is classified using Equation 4 into one of several tissue types, and together the entire image can be segmented. However, since the sample regions are relatively large in size, the boundary between segmented tissue layers is usually poorly resolved.

To refine the boundary between the tissue types, we use smaller window sizes. This produces overlapping windows and increases the computational requirement dramatically. Computational efficiency is improved through progressive refinement of the class boundaries. After a classification iteration, sample regions with different class neighbors are re-examined in the next iteration. After each iteration, the step size is reduced by half. Depending on the total image size, the minimum step size varies due to computation time constraint. While progressive boundary refinement improves efficiency, it may decrease sensitivity to small, isolated tissue regions.

\footnotetext{
${ }^{2}$ In the image analysis literature [3] it is common practice to assume feature independence and uniform distribution as an approximation if no more a priori knowledge is available about the features and their distribution.
} 


\subsection{Volume Rendering}

In this section we describe our method for combining the maximum probability classification described in Section 2.3 as well as the registration techniques presented in Section 2.2 to produce 3D volume renderings of the placenta dataset. Furthermore, we describe the issues associated with physical-level artifacts that make standard volume rendering techniques based on diffuse and specular lighting difficult to apply.

\subsubsection{Emissive Volume Rendering}

The microtome slicing of the placenta generates unavoidable and random shifts in distal maternal layers. Internal medial tissues are subtly shifted through stretching, squeezing, folding, or introduction of slice-wise discontinuous blood clots. Some of these shape and structure altering artifacts cannot be removed through registration, nor smoothing since our Bayesian classification scheme operates on a slice-by-slice basis. The result of this per-slice discontinuity causes the classification maps to be disjoint as well.

The implication for volume rendering is that smooth threedimensional boundaries cannot exist in the placenta data. Thus, diffuse and specular lighting across the rough three-dimensional probability surface only confuses the rendered images, rather than ascribing scope and depth. To address this issue we chose to volume render the data as an emissive volume only, thus smoothing the boundaries while still allowing the viewer to perceive depth in the three-dimensional tissue level regions. The implementation of the volume rendering algorithm used to generate the results in this paper is the standard volume rendering ray casting technique as implemented by VTK's vtkVolumeRayCastMapper class [5].

\subsubsection{Rendering Framework}

We take the results of the classification and map each pixel to a scalar value based on the class it belongs to. Furthermore we ensure that the scalar values for different classes are "far" apart so that boundaries between tissue types are well defined in the final volume render.

We assemble the volume by stacking the registered scalar mapped classifications onto each other, then treating the resulting data as a three-dimensional volume. Finally, we load the volume into Kitware's VolView [4] and select transfer functions to highlight desired classes. Since the class map is essentially a volume of intensities (see Figure 4), we select which class to render by adjusting the opacity and color for the particular intensity value that corresponds to the class of interest. The resulting image is a volume rendering of the segmentations of the volume, rather than the raw data directly [9].

\section{Results}

The datasets presented in this paper were harvested from either mutant mouse placenta $\left(R b^{-}\right)$, or wildtype mouse placenta, which was harvested at 13.5 days of gestation. The placenta was fixed in paraffin and sectioned at $5 \mu \mathrm{m}$ thickness using a microtome. Serial sections were mounted on glass slides and stained with hematoxylin and eosin. Finally, the slides were scanned in an Aperio ScanScope slide digitizer at $20 \times$ magnification. Each dataset on average contains 800 RGB slides whose average image dimensions are $15 \mathrm{~K} \times 15 \mathrm{~K}$ pixels. Thus, a single dataset measures 550 gigabytes in size. The volume of each placenta is approximately $1 \mathrm{~mm}^{3}$.

Although high resolution is necessary to observer cellular phenotypes, a lower resolution suffices for tissue level phenotypes. Thus for volume rendering we first manually remove the damaged slides and downsample the images to $500 \times 500$ using a Lanczos filter so that the volume fits into the memory of a single computer. The resulting slides are then classified into regions using the framework described in Section 2 and then registered using the framework described in Section 2.2.

Our goal is to highlight the tissue regions of labyrinth and intermixing of the labyrinth/spongiotrophoblast layers within the 3D data. In general we desire to see the overall three-dimensional structure of the placenta, as well as examine any infiltrations into the labyrinth layer such as the images in Figure 5 show. In both of these figures the volume is cropped to zoom in on the interesting features.

Figure 6 shows a comparison between three mouse placenta datasets we analyzed. Two are knockout $R b^{-}$while the third is a wildtype. The physiological differences between the placentas are noticeable as the roughness and comparative smoothness in the labyrinths between the specimens (column (b) is a wildtype placenta and its labyrinth layer (highlighted in blue) is quite smooth as compared to the $R b^{-}$placentas in columns (a) and (c)). Intermixing is highlighted in the third row by highlighting the areas which transition from labyrinth to any other material in yellow. We observe that the wildtype labyrinth layer's intermixing layer is mostly on the outer surface of the layer, while for the $R b^{-}$placentas intermixing occurs more freely throughout the layer.

These changes in the phenotype are consistent with our collaborators hypothesis that the absence of the retinoblastoma gene causes uncontrolled cell duplication in the placenta thus roughening the spongiotrophoblast-labyrinth interface. Aside from identifying these phenotype changes, a full three dimensional visualization has provided our collaborators with key insights to the overall spatial organization of the spongiotrophoblast-labyrinth interface.

\section{FUTURE WORK}

Overall, our results look promising, however the clarity of the rendering depends on registration and segmentation. Thus, tissue segmentation relating to accuracy and fine feature detection is still an outstanding issue in dealing with our $R b^{-}$mouse placenta data. Most of our future work will attempt to address these problems.

Our volume renderer classification scheme presented here was based directly on the results of maximum probability classification. We plan to generate transfer functions directly based on multivariate data generated from gradients of the densities of sub-tissue level features such as nuclei, redblood cell, and cytoplasm density. We expect that together the boundaries of these densities will highlight tissue level regions that could not be determined by a single feature alone.

Furthermore, we plan to examine continuous sub-tissue classification techniques. Rather than definitively classify a pixel as a certain sub-tissue feature (red blood cell, nuclei, cytoplasm, etc.) we will experiment with directly rendering the continuous probability for each sub-tissue feature type. We expect that boundary detection on such densities will yield smoother and more accurate boundaries than the discrete classification alone.

\section{Conclusions}

Volume visualization is more than simple volume rendering. In the real world case presented in this paper we required sophisticated registration techniques and two levels of segmentation before any visualization of 3D intermixing could occur.

Furthermore, we found the three-dimensional renderings useful for not only biological analysis but also to help drive the development of our pipeline; specifically,

- Volume renderings enhanced our understanding of the three dimensional structure of inter-placenta tissue types; this 

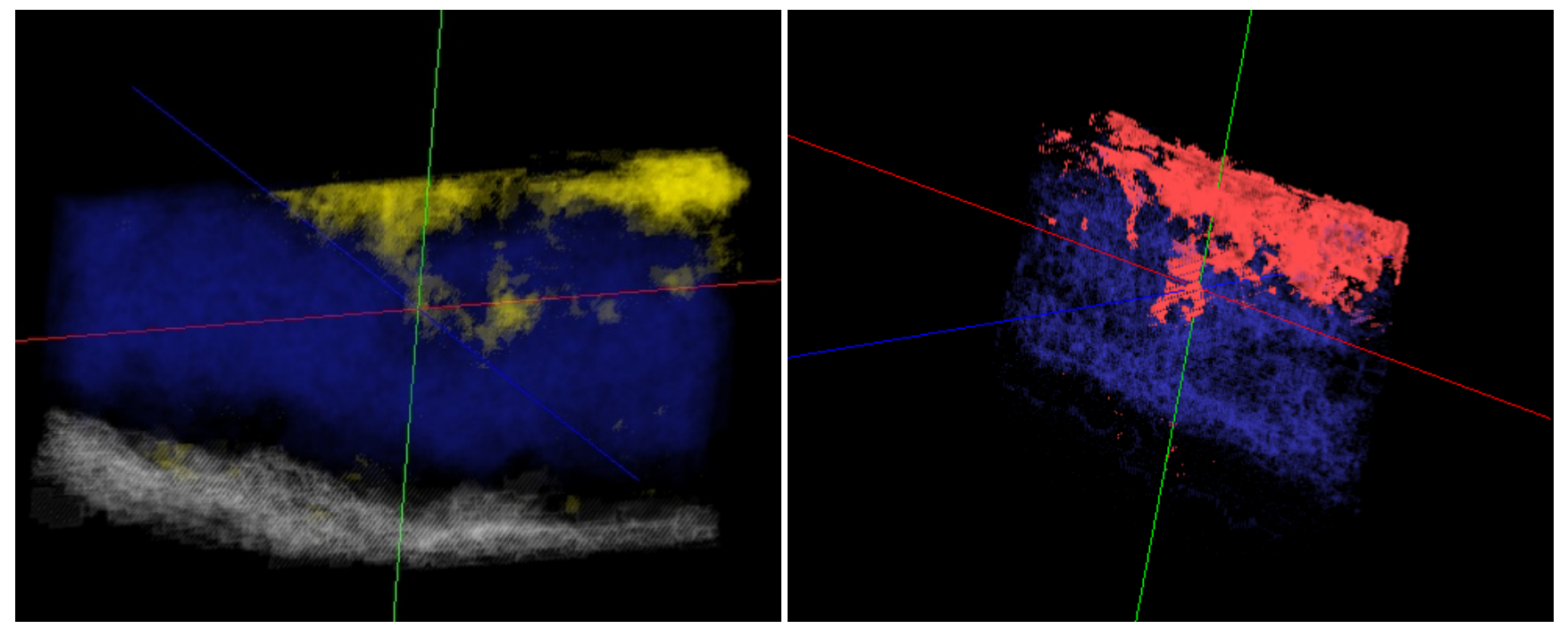

Figure 5: Both images are cropped volumes from the original data focusing on infiltrations into the labyrinth layer. In both images the labyrinth is shown in blue. In the left image spongiotrophoblast is shown in yellow and placenta boundary in white, while the right image highlights spongiotrophoblast in red. The transfer function sets the particular classified types to a high opacity while all other regions are set to zero. The cross-hairs indicate the center of the infiltration into the labyrinth layer.

would be extremely difficult to interpret given the original unregistered unclassified two dimensional histology slides.

- The three-dimensional visualization helped us understand where to improve registrations by locating problematic slices that caused the volume to "twist." It is otherwise very difficult to understand which slices can cause the registration pipeline to skew the volume.

- Although large "fingers" were visible in the volume, we could see that small "fingers" were lost to shear deformation. Using this knowledge we are exploring localized deformation models in registration, and also microtomed thicker slices in later specimens to reduce the shear.

In conclusion, volume visualization of our placenta data not only enhanced our ability to identify the biological features of the model, but also gave us valuable intuitive feedback on the effectiveness of our placenta processing pipeline.

\section{REFERENCES}

[1] I. Arganda-Carreras, R. Fernandez-Gonzalez, and C. Ortiz de Solorzano. Automatic registration of serial mammary gland sections. In Proceedings of the 26th Annual International Conference of the IEEE EMBS, September 2004.

[2] J. Clendenon, C. Phillips, R. Sandoval1, S. Fang, and K. Dunn. Voxx: a pc-based, near real-time volume rendering system for biological microscopy. American Journal of Physiology - Cell Physiology, 282(1):213-218, 2002.

[3] R. Duda, P. Hart, and D. Stork. Pattern Classification, volume 2. John Wiely \& Sons, Inc., 2001.

[4] Kitware Inc. VolView. http://www.kitware.com/products/volview.html

[5] Kitware Inc. VTK: the visualization toolkit. http://public.kitware.com/VTK.

[6] H. Johnson and G. Christensen. Consistent landmark and intensitybased image registration. In IEEE Transactions on Medical Imaging, volume 21, pages 450-461, 2002.

[7] A. Mojsilovic, B. Rogowitz, J. Gomes, and T. Deisboeck. Analysis and $3 \mathrm{~d}$ reconstruction of heterogeneity in malignant brain tumors:
An interdisciplinary case study using a novel computational visualization approach. Analytical and Quantitative Cytology and Histology, 24:125-133, June 2002

[8] Mouse Genome Sequencing Consortium. Initial sequencing and comparative analysis of the mouse genome. Nature, 420:520-562, 2002.

[9] K. Mueller, S. Lakare, and A. Kaufman. Scientific Visualization: Extracting Information and Knowledge from Scientific Data Sets, chapter Volume exploration made easy using feature maps. Springer-Verlag, Heidelberg, Germany, 2005.

[10] S. Ourselin, A. Roche, G. Susol, X. Pennec, , and N. Ayache. Reconstructing a $3 \mathrm{~d}$ structure from serial histological sections. Image and Vision Computing, 19(1):25-31, 2001.

[11] S. Petushi, C. Katsinis, C. Coward, A. Tozeren, and F. Garcia. Automated identification of microstructures on histology slides. In International Symposium on Biomedical Imaging, pages 424-427, 2004.

[12] K. Rao, T. Pan, R. Sharp, R. Ridgway, R. Machiraju, K. Huang, and J. Saltz. Registration and $3 \mathrm{~d}$ visualization of large microscopy images. In SPIE Symposium on Medical Imaging, 2006. To appear in proceedings.

[13] K. Huang T.C. Pan. Virtual mouse placenta: Tissue layer segmentation. Proceedings of the 27th Annual International Conference of the IEEE Engineering in Medicine and Biology Society (EMBC2005), 2005.

[14] M. Teverovskiy, V. Kumar, J. Ma, A. Kotsianti, D. Verbel, A. Tabesh, H-Y. Pang, Y. Vengrenyuk, S. Fogarasi, and O. Saidi. Improved prediction of prostate cancer recurrence based on an automated tissue image analysis system. In International Conference of Machine Learning Applications, pages 257-260, 2002.

[15] L. Wu, A. de Bruin, H. I. Saavedra, M. Starovic, A. Trimboli, Y. Yang, J. Opavska, P. Wilson, J. C. Thompson, M. C. Ostrowski, T. J. Rosol, L. A. Woollett, M. Weinstein, J. C. Cross, M. L. Robinson, and $\mathrm{G}$. Leone. Extra-embryonic function of $\mathrm{Rb}$ is essential for embryonic development and viability. Nature, 421:942-947, 2003. 
(a)
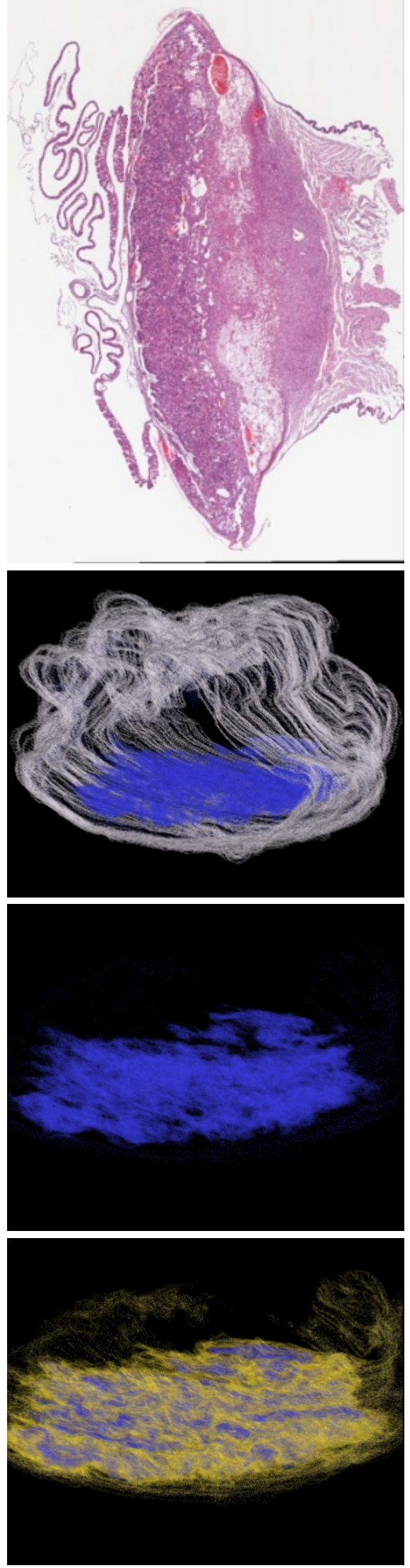

(b)
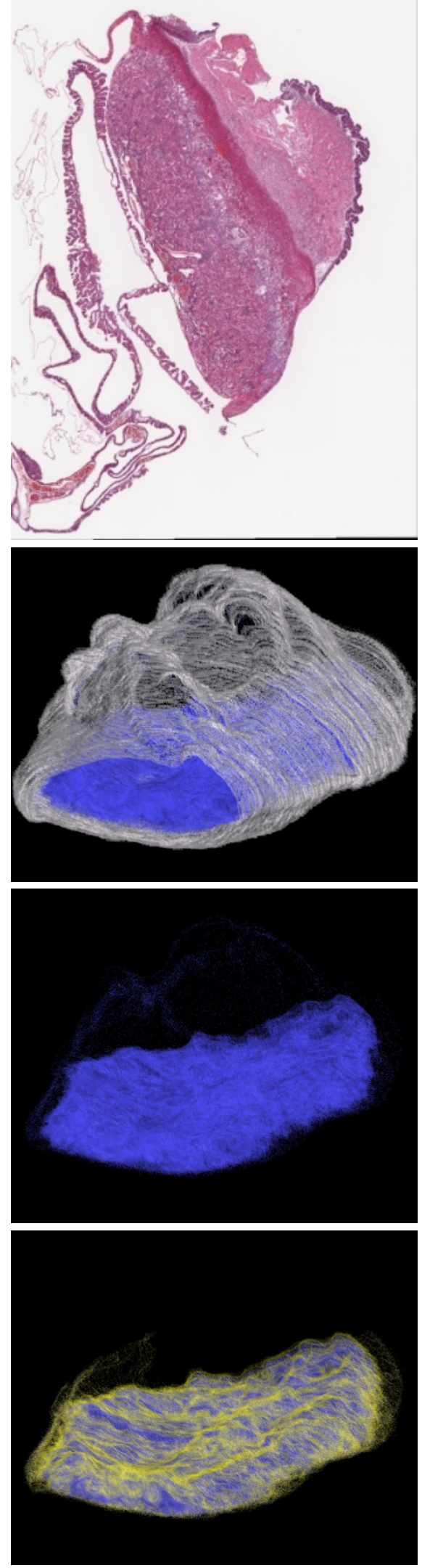

(c)
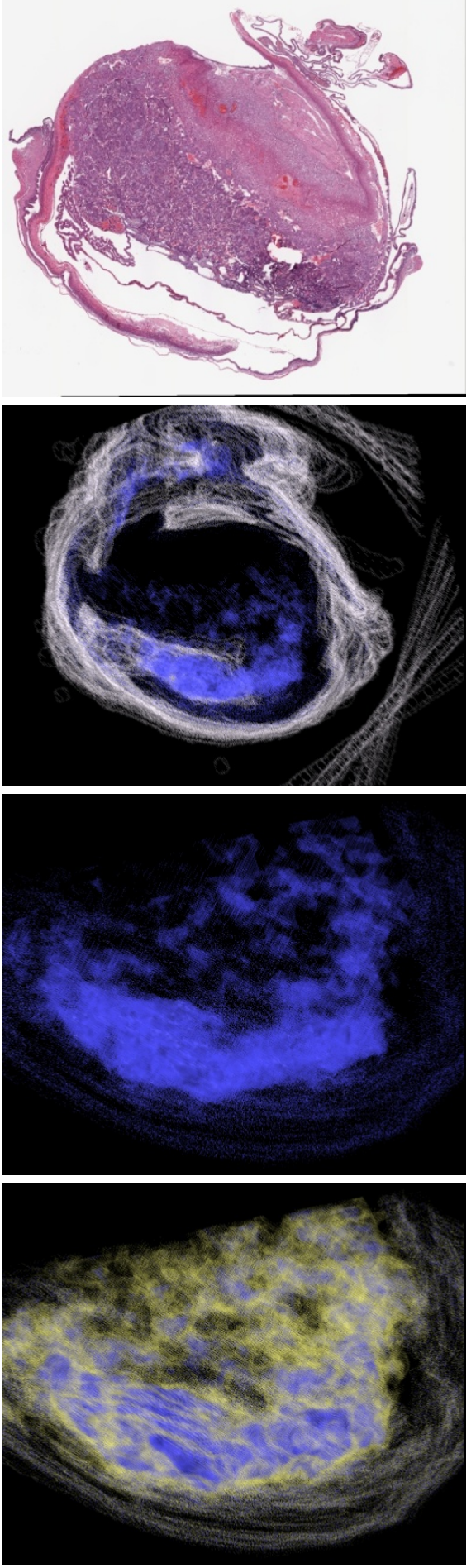

Figure 6: This figure highlights the difference between: (a) $R b^{-}$placenta, (b) wildtype $\left(R b^{+}\right.$) and (c) a noisy $R b^{-}$placenta. The first row shows a sample RGB slide from the dataset visualized in that column. The second row shows the overall 3D structure of the placenta with the outer layer in white and the labyrinth layer in blue. The third highlights only the labyrinth layer (notice the $R b^{-}$datasets have rougher labyrinths due to higher interdigitation between layers). This transfer function simply colors the volume blue at a fixed opacity when it encounters a labyrinth label. The final row highlights the intermixing of labyrinth and spongiotrophoblast layers by increasing in opacity and coloring yellow where the labyrinth label transitions into any other tissue type. 